\title{
Facilitative glucose transporter Glut1 is actively excluded from rod outer segments
}

\author{
Sidney M. Gospe, III', Sheila A. Baker ${ }^{2}$ and Vadim Y. Arshavsky ${ }^{1,2, *}$ \\ ${ }^{1}$ Department of Pharmacology and Cancer Biology and ${ }^{2}$ Department of Ophthalmology, Duke University School of Medicine, Durham, NC 27710, \\ USA \\ *Author for correspondence (vadim.arshavsky@ duke.edu)
}

Accepted 12 July 2010

Journal of Cell Science 123, 3639-3644

(C) 2010. Published by The Company of Biologists Ltd

doi:10.1242/jcs.072389

\section{Summary}

Photoreceptors are among the most metabolically active cells in the body, relying on both oxidative phosphorylation and glycolysis to satisfy their high energy needs. Local glycolysis is thought to be particularly crucial in supporting the function of the photoreceptor's light-sensitive outer segment compartment, which is devoid of mitochondria. Accordingly, it has been commonly accepted that the facilitative glucose transporter Glut1 responsible for glucose entry into photoreceptors is localized in part to the outer segment plasma membrane. However, we now demonstrate that Glut1 is entirely absent from the rod outer segment and is actively excluded from this compartment by targeting information present in its cytosolic C-terminal tail. Our data indicate that glucose metabolized in the outer segment must first enter through other parts of the photoreceptor cell. Consequently, the entire energy supply of the outer segment is dependent on diffusion of energy-rich substrates through the thin connecting cilium that links this compartment to the rest of the cell.

Key words: Glut1, Glucose transport, Photoreceptor

\section{Introduction}

The retina is among the most metabolically active vertebrate tissues. It is particularly susceptible to acute ischemic damage, and chronic impairment of nutrient delivery is thought to contribute to the progression of different degenerative retinal diseases (Punzo et al., 2009; Umino et al., 2006). The rod and cone photoreceptors have particularly strenuous energy requirements. In the dark, they expend substantial energy powering ion pumps to maintain both the circulating dark current and $\mathrm{Ca}^{2+}$ flux for synaptic transmission; when illuminated, photoreceptors need significant energy to perform visual signal transduction [see Okawa et al. (Okawa et al., 2008) for a recent detailed analysis of photoreceptor energy consumption]. Photoreceptors are highly polarized cells that sequester key biochemical reactions in anatomically distinct compartments. For example, the $\mathrm{Na}^{+} / \mathrm{K}^{+}$-ATPase, ultimately responsible for maintaining the dark current, is localized to the photoreceptor inner segment (Stahl and Baskin, 1984), where numerous mitochondria supply ATP through oxidative phosphorylation. Conversely, the energyconsuming reactions that occur in the light take place in the photoreceptor outer segment. Joined to the inner segment only by a thin connecting cilium, this apical compartment consists of a plasma membrane enclosing an ordered stack of hundreds of membrane discs. Because the outer segment is devoid of mitochondria, it has been proposed that the energy used in this compartment is supplied by the inner segment by diffusion of ATP through the connecting cilium (Linton et al., 2010). However, the presence of glycolytic enzymes in the outer segment, as determined by biochemical (Hsu and Molday, 1991; Hsu and Molday, 1994; Lowry et al., 1961; McConnell et al., 1969) and immunohistochemical (Hsu and Molday, 1991) methods, suggests that the outer segment is not entirely dependent on inner segment metabolism. Rather, it appears to possess the energy-generating capacity to meet at least some of its own needs through glycolysis.
Understanding how energy-rich substrates are delivered to the highly active photoreceptor cells has been of longstanding interest. Photoreceptors subjected to hypoglycemic conditions are known to have the capacity to take up and metabolize lactate (Winkler, 1981). A potential source of lactate is the neighboring Müller glial cell (Poitry-Yamate et al., 1995). However, there is controversy as to whether photoreceptors metabolize significant amounts of lactate under normal physiological conditions (Chih et al., 2001; Winkler et al., 2004; Acosta et al., 2005). The weight of the evidence appears to support the classic view that the major (if not sole) photoreceptor energy substrate in vivo is glucose (Cohen and Noell, 1960). The delivery of glucose to photoreceptors is crucially dependent on Glut1, the most ubiquitously expressed member of the GLUT family of facilitative glucose transporters. Glut1 has two roles in this process. The first is to transport glucose from the choroidal vasculature to the outer retina across the blood-retina barrier formed by the retinal pigment epithelium (RPE). Both the apical and basolateral membranes of RPE cells contain Glut1 (Takata et al., 1990; Takata et al., 1992), allowing direct passage of glucose down its concentration gradient to the retina. The second role of Glut1 is to mediate glucose uptake by the photoreceptors themselves, because it is the only known glucose transporter expressed by these cells (Mantych et al., 1993). For nearly 20 years, Glut1 has been thought to localize in part to the plasma membrane of photoreceptor outer segments, on the basis of glucose transport activity measured in bovine rod outer segment preparations (Hsu and Molday, 1991; Lopez-Escalera et al., 1991) and immunohistochemical data published by a number of laboratories (Elliott et al., 2008; Hsu and Molday, 1991; Mantych et al., 1993; Nihira et al., 1995) (but see Kumagai et al., 1994). The presence of Glut1 on the outer segment plasma membrane has stood as an additional argument for local glucose utilization by the outer segment. 
Here, we report new evidence that contradicts the notion that glucose can directly enter the outer segment through Glut1. Rather, we find that Glut 1 is actively excluded from the outer segment by the photoreceptor protein sorting machinery. We arrived at this conclusion using several independent approaches: immunofluorescence, immuno-electron microscopy and transgenic expression of GFP-labeled Glut1 constructs. Therefore, for glucose to be used locally in the outer segment, it must diffuse there after first entering the photoreceptor via Glut1 in the inner segment.

\section{Results and Discussion}

\section{Glut1 localization in the mammalian retina}

Immunostaining of Glut1 in the rat retina produced a localization pattern that was largely similar to previous studies conducted in mammals (Fig. 1). Glut1 is expressed in the ganglion cell layer, in the neuronal processes of both plexiform layers and in the cell bodies of the inner nuclear layer. Within photoreceptors, it was detectable from the inner segments through the outer nuclear layer to the synaptic terminals. The highest expression of Glut1, however, was not observed in the neural retina, but in the apical and basolateral membranes of the overlying RPE. Notably, Glut1 was also observed in linear arrays descending from the RPE about halfway down the length of the outer segments. Importantly, these arrays were continuous with the intensely stained RPE apical membrane and did not reach the junction between the outer and inner segments. These features are not consistent with staining of the outer segment plasma membrane; instead, they are characteristic of RPE microvilli that interdigitate between neighboring photoreceptor outer segments (Dowling and Gibbons, 1962). Our observation suggests that outer segments either contain much less Glut1 than other photoreceptor compartments or do not contain Glut1 at all, leading us to investigate this unexpected finding in further detail.

\section{Glut1 localization in the amphibian retina}

Mammalian retina is not an optimal model for histological delineation of proteins between RPE microvilli and outer segments, owing to the relatively short length of the outer segments $(\sim 25 \mu \mathrm{m})$

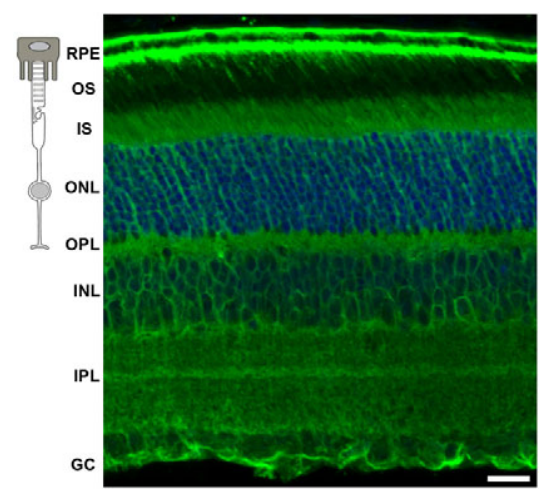

Fig. 1. Glut1 immunolocalization in mammalian retina. A rat retina section immunostained for Glut1 (green). Nuclei are counter-stained with Hoechst 33342 (blue). To the left is a schematic of a rat rod photoreceptor with an overlying RPE cell, illustrating the relative positions of individual subcellular compartments. RPE, retinal pigment epithelium; OS, outer segment; IS, inner segment; ONL, outer nuclear layer; OPL, outer plexiform layer; INL, inner nuclear layer; IPL, inner plexiform layer; GC, ganglion cell layer. Scale bar: $20 \mu \mathrm{m}$. and their small diameter $(\sim 1.5 \mu \mathrm{m})$. Therefore, we decided to continue our investigation in Xenopus laevis, which offers both larger rod outer segments $(\sim 80 \times 6 \mu \mathrm{m})$ for easier anatomic distinction and the opportunity to generate transgenic animals for in vivo experiments very rapidly. Because commercially available antibodies against mammalian Glut1 do not react against the Xenopus isoform (Fig. 2A), we generated an affinity-purified polyclonal antibody against the $15 \mathrm{C}$-terminal amino acids of Xenopus Glut1, which specifically recognized a band migrating $\sim 47 \mathrm{kDa}$ in a Xenopus retinal extract (Fig. 2B). Immunolabeling of Glut1 in Xenopus retinal cryosections revealed a similar localization pattern to that in the rat (Fig. 2C). Glut1 was found in the ganglion cell layer and exhibited weak and diffuse expression in the inner plexiform layer and inner nuclear layer. Within the prominent rod photoreceptors, Glut1 stained brightly in the vicinity of the synapses and around the cell bodies located in the outer nuclear layer. There was also distinct (although weaker) signal in the rod inner segments and in their calycal processes, which are inner segment protrusions extending partway up the base of the outer segment (indicated with arrowheads in Fig. 2D-F). The inner-segment-specific Glut1 staining colocalized with the $\mathrm{Na}^{+} / \mathrm{K}^{+}$-ATPase (Fig. 2E,F), which strongly labels the inner segment plasma membrane and is entirely absent from outer segments (Spencer et al., 1988; Stahl and Baskin, 1984). The most intense Glut1 signal was again seen in the RPE plasma membrane and apical microvilli, which extend roughly three-quarters of the way down the rod outer segments, but do not reach the outer-inner segment junction. Co-staining the cryosections with phalloidin confirmed the extensive colocalization of Glut1 and the actin-based RPE microvilli (and again the calycal processes; Fig. 2G-I).

To determine whether the microvilli and calycal processes mask the presence of less-abundant Glut1 in the plasma membrane of the outer segment, we isolated fragments of Xenopus rods containing intact outer segments with large portions of inner segment still attached. As shown in Fig. 2J-L, Glut1 was only found in the inner segment membranes and calycal processes but was not detected along the outer segment plasma membrane. This Glut1 localization pattern was indistinguishable between dark- and light-adapted animals (data not shown).

Our immunofluorescence observations were further supported by immuno-electron microscopy analysis. Immunogold labeling of longitudinal sections through Xenopus retina revealed that Glut1 is present in the thin RPE microvilli located to the sides of the rod outer segments (Fig. 3A). Rare gold particles occasionally found within the outer segments showed no predilection for the plasma membrane and were observed with similar frequency in sections incubated with control rabbit IgG (Fig. 3B), thus probably representing nonspecific background labeling. Cross-sections made through retinal flat mounts produced similar results, with clear labeling of RPE microvilli but no notable labeling of the rod outer segments above background levels (Fig. 3C,D). Quantification of the gold particle density within the samples confirmed the specificity of Glut1 immunolabeling of the microvilli but not of the outer segments (Fig. 3E). Taken together, these data strongly suggest that rod outer segments contain little, if any, Glut1.

\section{Active exclusion of Glut1 from rod outer segments of transgenic Xenopus tadpoles}

As a decisive test of our interpretation that Glut1 is not present in the outer segment, we overexpressed a GFP-Glut1 fusion protein 
A

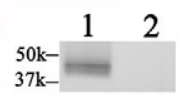

B

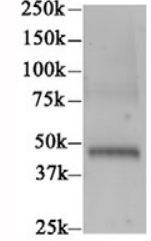

Glut1

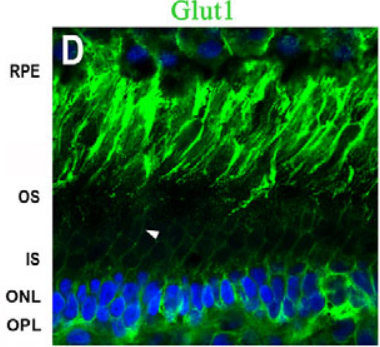

Glut1

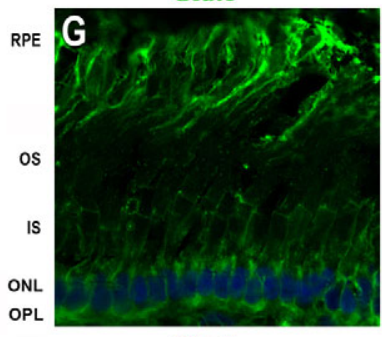

Glut1

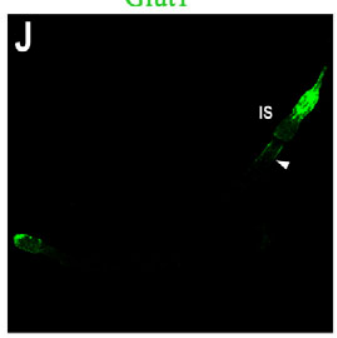

C

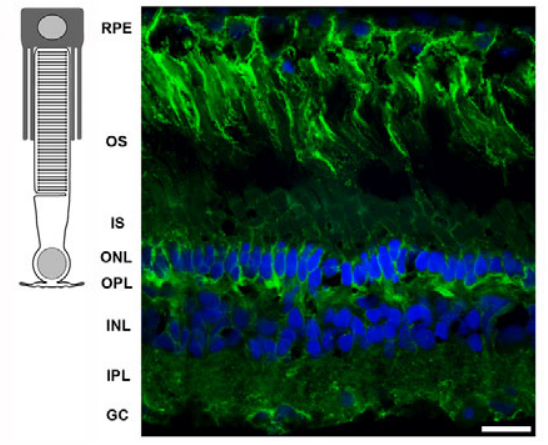

NKA

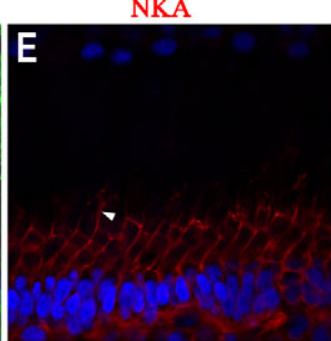
Phalloidin

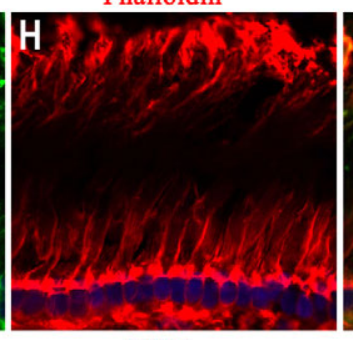

WGA

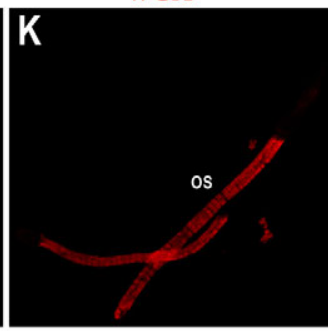

Merge

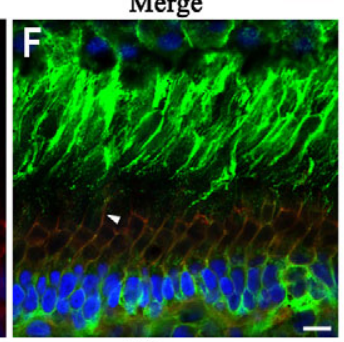

Merge

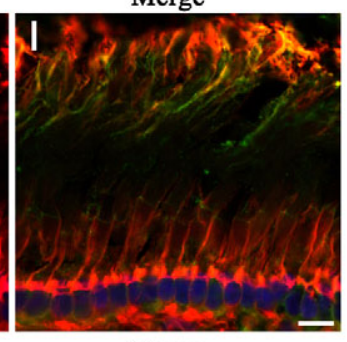

Merge

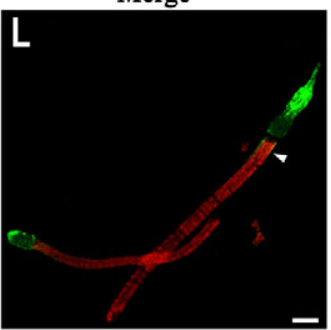

Fig. 2. Glut1 immunolocalization in amphibian retina.

(A) Western blot analysis of retinal extracts from mouse (lane 1) and Xenopus (lane 2), probed with anti-human Glut1 polyclonal antibody. The Xenopus isoform was not recognized. (B) The polyclonal antibody raised against Xenopus Glut1 specifically recognized this protein in an immunoblot of Xenopus retinal extract. (C) A section of Xenopus retina immunostained for Glut1 (green). Nuclei were counter-stained with Hoechst 33342 (blue). To the left is a schematic of a frog rod photoreceptor with an overlying RPE cell, illustrating the relative positions of individual subcellular compartments. Abbreviations are the same as in Fig. 1. Scale bar: $20 \mu \mathrm{m}$. (D-I) Xenopus retinal cryosections stained for Glut1 (green) and $\mathrm{Na}^{+} / \mathrm{K}^{+}$-ATPase (NKA, red; D-F) or phalloidin (red; G-I). Glut1 localized to the RPE and its microvilli, and to the rod photoreceptor plasma membrane in the synaptic, nuclear and inner segment layers. Labeling of the $\mathrm{Na}^{+} / \mathrm{K}^{+}$-ATPase (D-F) was used as a marker for the photoreceptor inner segment plasma membrane and calycal processes (arrowheads). Labeling of $\mathrm{F}$-actin with phalloidin (G-I) was used as a marker for the RPE microvilli, in addition to the photoreceptor calycal processes. (J-L) Staining of two isolated rod photoreceptor fragments revealed Glut1 (green) on inner segment (IS) membranes and calycal processes

(arrowheads) but not along the plasma membrane of outer segments (OS) marked by wheat germ agglutinin (WGA, red). Scale bars: $10 \mu \mathrm{m}(\mathrm{D}-\mathrm{L})$. in the rods of transgenic Xenopus tadpoles (Fig. 4A,B). GFP signal was prominent in the entire rod plasma membrane, except for that of the outer segment. The calycal processes lining the base of the outer segment were particularly well visualized in this case. Notably, the signal did not extend along the entire length of the outer segment, just as expected for a protein actively excluded from this compartment by the intracellular protein sorting machinery. This experiment ruled out any immunostaining artifact that might have accounted for our failure to observe endogenous Glut1 in the outer segment.

It is well established that the plasma membrane of the photoreceptor cell, although electrically continuous, is separated into two functional domains characterized by distinct protein compositions (for a review, see Insinna and Besharse, 2008). The first domain surrounds the outer segment and the second encloses the rest of the cell, with a protein diffusion barrier located near the connecting cilium that joins the inner and the outer segments. Previous work in our laboratory has demonstrated that the membrane-rich outer segment is a preferred destination for membrane proteins heterologously expressed in tadpole rods and that exclusion of these proteins from the outer segment requires the presence of intrinsic targeting information to specify localization elsewhere in the cell (Baker et al., 2008). Because the cytosolic Cterminal tail of Glut1 has been implicated in the regulation of its intracellular trafficking in other cell types (Verhey et al., 1993; Wieman et al., 2009), we generated transgenic tadpoles expressing a truncated Glut1 construct (GFP-Glut1 $\Delta 38)$ lacking the entire C terminus. Strikingly, although this truncation did not prevent the protein from reaching the synaptic terminal and inner segment plasma membrane, it did allow the construct to gain entry into the rod outer segment, including the disc membranes (Fig. 4C). These data not only confirm that the full-length Glut1 protein localizes outside of the outer segment, but also demonstrate that photoreceptors use a targeting signal within the C-terminal tail of Glut1 to actively restrict it from this domain.

\section{Concluding remarks}

In summary, our observations refute the long-held idea that Glut1 exists in the rod outer segment plasma membrane to allow direct entry of glucose into this compartment. Such a role for Glut1 was 


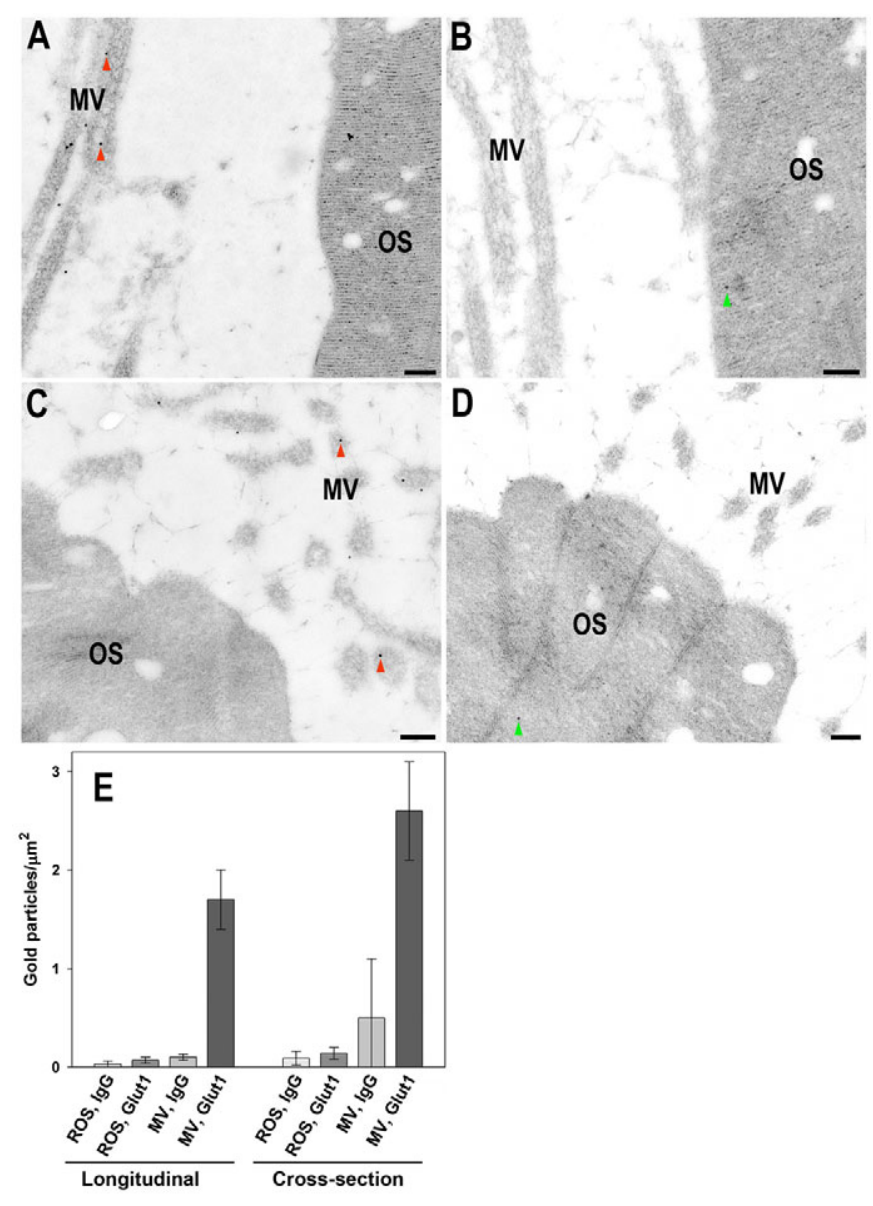

first proposed based on experimental evidence that glucose enters purified bovine rod outer segments in a process that can be inhibited by cytochalasin B and phloretin, both known antagonists of transport by the GLUT family of glucose transporters (Hsu and Molday, 1991; Lopez-Escalera et al., 1991). Immunohistochemical evidence of Glut1 in the outer segments from a variety of species has been offered by a number of laboratories in support of this model, but in all cases the resolution of the images did not allow outer segment plasma membranes to be distinguished from RPE microvilli (Elliott et al., 2008; Hsu and Molday, 1991; Mantych et al., 1993; Nihira et al., 1995). No other glucose transporters have
Fig. 3. Immunogold analysis of Glut1 in Xenopus retina sections. Longitudinal sections $(\mathbf{A}, \mathbf{B})$ and cross-sections $(\mathbf{C}, \mathbf{D})$ through Xenopus retina were processed for immunogold analysis. Gold particles (representatives marked by red arrowheads) were abundant on the fingerlike RPE microvilli (A) and their cross-sections (C). Rare, nonspecific particles (green arrowheads) were occasionally found within the rod outer segments, with no preference for the lateral plasma membrane; they were found at similar frequencies in control sections incubated with rabbit IgG control (B,D). (E) Gold particle labeling densities (particles per $\mu \mathrm{m}^{2}$ of membrane) were calculated for the outer segments and microvilli in both Glut1-stained and control sections. The mean labeling density for each type of structure in ten separate images is plotted with error bars representing 95\% confidence intervals. MV, microvilli; OS, outer segment; ROS, rod outer segment. Scale bars: $200 \mathrm{~nm}$.

been identified in the outer segment region in immunohistochemical experiments (Mantych et al., 1993) or in proteomic analyses (Kwok et al., 2008; Liu et al., 2007). Therefore, based on our data, we suggest that the original biochemical evidence of direct glucose entry into purified outer segment preparations reflects the presence of Glut1 in contaminating membranes from the RPE or inner segments. Alternatively, mechanical shearing of the outer segments during their isolation might have destroyed the diffusion barrier at the connecting cilium, allowing the compositions of the outer segment plasma membrane and the cell body to become mixed (e.g. Spencer et al., 1988).
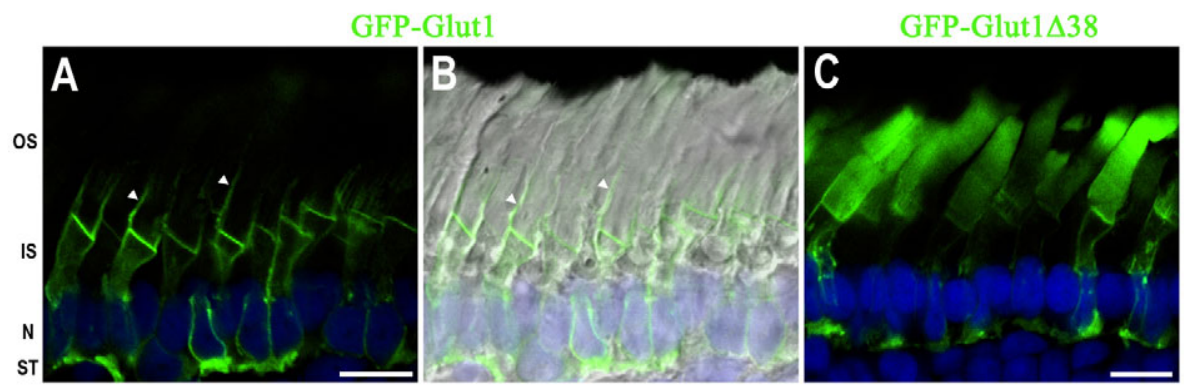

Fig. 4. GFP-tagged Glut1 is actively excluded from rod outer segments of transgenic Xenopus tadpoles. (A,B) GFP-tagged Glut1 was expressed in transgenic tadpole rods and localized to the plasma membrane of the synaptic terminal, nuclear region and inner segment, including the calycal processes (arrowheads). The outer segments extend well beyond the GFP signal in the calycal processes (B). (C) GFP-tagged Glut1 mutant lacking its 38-residue C-terminal tail (GFPGlut1 $\Delta 38$ ) displayed prominent outer segment localization in tadpole rods. OS, outer segment; IS, inner segment; N, nucleus; ST, synaptic terminal. Scale bars: $10 \mu \mathrm{m}$. 
We should emphasize that our findings do not invalidate the concept of glucose utilization in the outer segment. Rather, they suggest that glucose supplied to the retina by the RPE first enters photoreceptor cells through Glut1 located in the inner segment plasma membrane. Indeed, this is an attractive idea because hexokinase (the enzyme that mediates the first reaction in glucose metabolism by producing glucose-6-phosphate) is confined to the inner segment (Aslanukov et al., 2006; Wilkin and Wilson, 1977), presumably owing to association with outer mitochondrial membranes (for a review, see Mathupala et al., 2009). Glucose phosphorylation by hexokinase is required to trap glucose inside the cell because glucose-6-phosphate cannot escape the cell through glucose transporters. Therefore, direct glucose entry into the outer segment compartment devoid of hexokinase would be unproductive. Instead, glucose-6-phosphate produced by hexokinase in the inner segment can undergo further metabolism in both the inner and outer segments. To reach the outer segment it must diffuse through the connecting cilium, which therefore serves as the sole route for delivering energy-rich substrates to this compartment. Once in the outer segment, glucose-6-phosphate can either fuel glycolysis to produce ATP required for visual signal transduction (Hsu and Molday, 1994; Okawa et al., 2008) or enter the pentose phosphate pathway (Futterman et al., 1970; Hsu and Molday, 1994), which produces NADPH required to initiate regeneration of visual pigment bleached by light.

Finally, our observation that Glut1 is actively excluded from the outer segment necessitates revision of a short list of outer segment proteins believed to be restricted to the plasma membrane and kept out of discs. This leaves only the cyclic nucleotide-gated channel (Cook et al., 1989), the $\mathrm{Na}^{+} / \mathrm{Ca}^{2+} / \mathrm{K}^{+}$exchanger (Reid et al., 1990) and the insulin receptor (Rajala et al., 2007) as outer segment proteins with documented plasma-membrane-specific localization. With regard to the targeting of Glut1, our data raise an interesting question: what targeting mechanism is responsible for its exclusion from the outer segment? We have shown that the 38-residue cytosolic tail of Glut1 is necessary for this localization pattern. The last four amino acids (DSQV) constitute a PDZ-binding motif that is necessary for an interaction with the protein GIPC (Bunn et al., 1999), which has been shown to regulate recycling of internalized Glut1 to the plasma membrane in hematopoietic cells (Wieman et al., 2009). Two other proteins, the small ubiquitin-like modifier (SUMO)-conjugating enzyme Ubc9 (Giorgino et al., 2000) and the lipid raft protein stomatin (Zhang et al., 2001; Zhang et al., 1999), have also been reported to associate with the cytosolic tail of Glut1. Whether any of these proteins are expressed in photoreceptors and have a role in establishing the localization pattern of Glut1 awaits further investigation.

\section{Materials and Methods}

\section{Antibody production}

Polyclonal anti-peptide antibody against Xenopus laevis Glut1 was generated in rabbits at the Duke University animal facility using the peptide CAEELNTFGHADDSQV conjugated to keyhole limpet hemocyanin (KLH). Affinity purification of the antibody from rabbit serum was achieved using the same peptide coupled to the Sulfolink Plus column (Pierce Biotechnology), following the manufacturer's instructions.

\section{Western blot analysis}

Retinas isolated from adult Xenopus laevis or CD-1 mice were solubilized in SDSPAGE sample buffer, sonicated and boiled. Proteins were separated on 4-20\% SDSPAGE gels, transferred onto polyvinylidine fluoride (PVDF) membranes and blotted with rabbit anti-human Glut1 (Abcam) or our polyclonal anti-Xenopus Glut1 antibody. Goat anti-rabbit Alexa Fluor 680 (Invitrogen) was used as a secondary antibody, and the blots were imaged using an Odyssey imaging system (LiCor).

\section{Production of transgenic tadpoles}

The N-terminal GFP-tagged rat Glut1 construct, a kind gift from J. Rathmell (Duke University, Durham, NC), was subcloned behind a $1.5 \mathrm{~kb}$ version of the Xenopus opsin promoter. The deletion construct GFP-Glut $1 \Delta 38$ was generated using a reverse PCR primer to add a STOP codon after Glu-454. Both constructs were fully sequence verified. Transgenic Xenopus laevis tadpoles were generated using the restriction enzyme-mediated integration method (Amaya and Kroll, 1999; Kroll and Amaya, 1996), modified as described in Baker et al. (Baker et al., 2008). Briefly, linearized plasmid DNA containing the transgene was mixed with Xenopus sperm nuclei in the presence of the restriction enzyme XhoI to promote integration. Transgenic sperm nuclei were then injected into Xenopus oocytes, and the resulting embryos were allowed to develop until stage 43-54. The retinas from a minimum of five positive transgenic animals were analyzed for each DNA construct.

\section{Immunofluorescence}

Posterior eyecups from rat eyes were obtained by microdissection, fixed in $4 \%$ paraformaldehyde for 1 hour, rinsed three times in PBS and embedded in $4.5 \%$ agarose (Invitrogen). 150- $\mu \mathrm{m}$ cross-sections through the central retina were collected using a vibratome (Leica VT1200S) in 24-well plates, and floating sections were blocked in $5 \%$ goat serum in the presence of $0.5 \%$ Triton X-100, incubated overnight with rabbit anti-human Glut1 diluted in blocker, and stained with goat anti-rabbit Alexa Fluor 488 secondary antibody (Invitrogen) and $10 \mu \mathrm{g} / \mathrm{ml}$ Hoechst 33342 (Invitrogen) to label nuclei.

Transgenic Xenopus tadpoles, adult Xenopus retinas and isolated photoreceptors were prepared as previously described (Baker et al., 2008). Prior to isolation of photoreceptor cells, frogs were dark adapted for 2 hours to promote RPE retraction. The following antibodies and stains were used: our polyclonal rabbit anti-Xenopus Glut1; monoclonal mouse anti- $\mathrm{Na}^{+} / \mathrm{K}^{+}$-ATPase alpha subunit (Santa Cruz Biotechnology, sc-58628); goat secondary antibodies conjugated to Alexa Fluor 488 or 594 (Invitrogen); phalloidin conjugated to Alexa Fluor 594 (Invitrogen) to label filamentous actin; and wheat germ agglutinin conjugated to Alexa Fluor 594 (Invitrogen) to label outer segments.

All samples were mounted with Fluoromount (Electron Microscopy Sciences, Hatfield, PA) under glass coverslips and visualized using a Nikon Eclipse 90i microscope and a $\mathrm{C} 1$ confocal scanner controlled by EZ-C1 v 3.10 software (Nikon).

\section{Immunogold analysis}

Adult Xenopus eyecups were fixed in $2 \%$ paraformadehyde and $0.5 \%$ glutaraldehyde for 3 hours, dehydrated and embedded in LR white (Ted Pella, Redding, CA). 65$\mathrm{nm}$ sections were stained with rabbit anti-Xenopus Glut1 in the presence of $2 \%$ goat serum and $0.1 \%$ saponin. Goat-anti-rabbit secondary antibody conjugated to $15 \mathrm{~nm}$ gold particles (Electron Microscopy Sciences) was then applied. In control experiments, sections were incubated with a rabbit IgG control (Abcam). Transmission electron microscopy was performed with a JEM-1400 instrument (JEOL, Tokyo, Japan).

Ten images at magnifications of $50,000 \times$ were taken of the longitudinal and cross-sections under both labeling conditions. The number of gold particles localized to rod outer segments or RPE microvilli were counted manually for each image. Gold particle counts were normalized to the total membrane area for each type of structure, which was measured using MetaMorph software (version 6.3r, Molecular Devices), and $95 \%$ confidence intervals for the mean gold particle labeling densities were constructed using StatView v 5.0.1 software (SAS Institute).

We thank Ying Hao for technical assistance with immunogold analysis and Boris Reidel for helpful discussion. The work was supported by NIH grant EY12859 (V.Y.A.) and Core Grant for Vision Research to Duke University EY5722. Deposited in PMC for release after 12 months.

\section{References}

Acosta, M. L., Fletcher, E. L., Azizoglu, S., Foster, L. E., Farber, D. B. and Kalloniatis, M. (2005). Early markers of retinal degeneration in $\mathrm{rd} / \mathrm{rd}$ mice. Mol. Vis. 11, 717-728. Amaya, E. and Kroll, K. L. (1999). A method for generating transgenic frog embryos. Methods Mol. Biol. 97, 393-414.

Aslanukov, A., Bhowmick, R., Guruju, M., Oswald, J., Raz, D., Bush, R. A., Sieving, P. A., Lu, X., Bock, C. B. and Ferreira, P. A. (2006). RanBP2 modulates Cox11 and hexokinase I activities and haploinsufficiency of RanBP2 causes deficits in glucose metabolism. PLoS Genet. 2, e177.

Baker, S. A., Haeri, M., Yoo, P., Gospe, S. M., 3rd, Skiba, N. P., Knox, B. E. and Arshavsky, V. Y. (2008). The outer segment serves as a default destination for the trafficking of membrane proteins in photoreceptors. J. Cell Biol. 183, 485-498.

Bunn, R. C., Jensen, M. A. and Reed, B. C. (1999). Protein interactions with the glucose transporter binding protein GLUT1CBP that provide a link between GLUT1 and the cytoskeleton. Mol. Biol. Cell 10, 819-832.

Chih, C. P., Lipton, P. and Roberts, E. L., Jr (2001). Do active cerebral neurons really use lactate rather than glucose? Trends Neurosci. 24, 573-578.

Cohen, L. H. and Noell, W. K. (1960). Glucose catabolism of rabbit retina before and after development of visual function. J. Neurochem. 5, 253-276. 
Cook, N. J., Molday, L. L., Reid, D., Kaupp, U. B. and Molday, R. S. (1989). The cGMP-gated channel of bovine rod photoreceptors is localized exclusively in the plasma membrane. J. Biol. Chem. 264, 6996-6999.

Dowling, J. E. and Gibbons, I. R. (1962). The fine structure of the pigment epithelium in the albino rat. J. Cell Biol. 14, 459-474.

Elliott, M. H., Nash, Z. A., Takemori, N., Fliesler, S. J., McClellan, M. E. and Naash, M. I. (2008). Differential distribution of proteins and lipids in detergent-resistant and detergent-soluble domains in rod outer segment plasma membranes and disks. $J$. Neurochem. 104, 336-352.

Futterman, S., Hendrickson, A., Bishop, P. E., Rollins, M. H. and Vacano, E. (1970). Metabolism of glucose and reduction of retinaldehyde in retinal photoreceptors. $J$. Neurochem. 17, 149-156.

Giorgino, F., de Robertis, O., Laviola, L., Montrone, C., Perrini, S., McCowen, K. C. and Smith, R. J. (2000). The sentrin-conjugating enzyme mUbc9 interacts with GLUT4 and GLUT1 glucose transporters and regulates transporter levels in skeletal muscle cells. Proc. Natl. Acad. Sci. USA 97, 1125-1130.

Hsu, S. C. and Molday, R. S. (1991). Glycolytic enzymes and a GLUT-1 glucose transporter in the outer segments of rod and cone photoreceptor cells. J. Biol. Chem. 266, 21745-21752.

Hsu, S. C. and Molday, R. S. (1994). Glucose metabolism in photoreceptor outer segments. Its role in phototransduction and in NADPH-requiring reactions. J. Biol. Chem. 269, 17954-17959.

Insinna, C. and Besharse, J. C. (2008). Intraflagellar transport and the sensory outer segment of vertebrate photoreceptors. Dev. Dyn. 237, 1982-1992.

Kroll, K. L. and Amaya, E. (1996). Transgenic Xenopus embryos from sperm nuclear transplantations reveal FGF signaling requirements during gastrulation. Development 122, 3173-3183.

Kumagai, A. K., Glasgow, B. J. and Pardridge, W. M. (1994). GLUT1 glucose transporter expression in the diabetic and nondiabetic human eye. Invest. Ophthalmol. Vis. Sci. 35, 2887-2894.

Kwok, M. C., Holopainen, J. M., Molday, L. L., Foster, L. J. and Molday, R. S. (2008). Proteomics of photoreceptor outer segments identifies a subset of SNARE and Rab proteins implicated in membrane vesicle trafficking and fusion. Mol. Cell. Proteomics 7, 1053-1066.

Linton, J. D., Holzhausen, L. C., Babai, N., Song, H., Miyagishima, K. J., Stearns, G. W., Lindsay, K., Wei, J., Chertov, A. O., Peters, T. A. et al. (2010). Flow of energy in the outer retina in darkness and in light. Proc. Natl. Acad. Sci. USA 107, 8599-8604.

Liu, Q., Tan, G., Levenkova, N., Li, T., Pugh, E. N., Jr, Rux, J. J., Speicher, D. W. and Pierce, E. A. (2007). The proteome of the mouse photoreceptor sensory cilium complex. Mol. Cell. Proteomics 6, 1299-1317.

Lopez-Escalera, R., Li, X. B., Szerencsei, R. T. and Schnetkamp, P. P. (1991). Glycolysis and glucose uptake in intact outer segments isolated from bovine retinal rods. Biochemistry 30, 8970-8976.

Lowry, O. H., Roberts, N. R., Schulz, D. W., Clow, J. E. and Clark, J. R. (1961). Quantitative histochemistry of retina. II. Enzymes of glucose metabolism. J. Biol. Chem. 236, 2813-2820.

Mantych, G. J., Hageman, G. S. and Devaskar, S. U. (1993). Characterization of glucose transporter isoforms in the adult and developing human eye. Endocrinology 133, 600-607.

Mathupala, S. P., Ko, Y. H. and Pedersen, P. L. (2009). Hexokinase-2 bound to mitochondria: cancer's stygian link to the "Warburg Effect" and a pivotal target for effective therapy. Semin. Cancer Biol. 19, 17-24.

McConnell, D. G., Ozga, G. W. and Solze, D. A. (1969). Evidence for glycolysis in bovine retinal microsomes and photoreceptor outer segments. Biochim. Biophys. Acta 184, 11-28.
Nihira, M., Anderson, K., Gorin, F. A. and Burns, M. S. (1995). Primate rod and cone photoreceptors may differ in glucose accessibility. Invest. Ophthalmol. Vis. Sci. 36, 1259-1270.

Okawa, H., Sampath, A. P., Laughlin, S. B. and Fain, G. L. (2008). ATP consumption by mammalian rod photoreceptors in darkness and in light. Curr. Biol. 18, 19171921.

Poitry-Yamate, C. L., Poitry, S. and Tsacopoulos, M. (1995). Lactate released by Muller glial cells is metabolized by photoreceptors from mammalian retina. J. Neurosci. 15, 5179-5191.

Punzo, C., Kornacker, K. and Cepko, C. L. (2009). Stimulation of the insulin/mTOR pathway delays cone death in a mouse model of retinitis pigmentosa. Nat. Neurosci. 12, 44-52.

Rajala, A., Anderson, R. E., Ma, J. X., Lem, J., Al-Ubaidi, M. R. and Rajala, R. V. (2007). G-protein-coupled receptor rhodopsin regulates the phosphorylation of retinal insulin receptor. J. Biol. Chem. 282, 9865-9873.

Reid, D. M., Friedel, U., Molday, R. S. and Cook, N. J. (1990). Identification of the sodium-calcium exchanger as the major ricin-binding glycoprotein of bovine rod outer segments and its localization to the plasma membrane. Biochemistry 29, 16011607.

Spencer, M., Detwiler, P. B. and Bunt-Milam, A. H. (1988). Distribution of membrane proteins in mechanically dissociated retinal rods. Invest. Ophthalmol. Vis. Sci. 29, 10121020 .

Stahl, W. L. and Baskin, D. G. (1984). Immunocytochemical localization of $\mathrm{Na}+, \mathrm{K}+$ adenosine triphosphatase in the rat retina. J. Histochem. Cytochem. 32, 248250 .

Takata, K., Kasahara, T., Kasahara, M., Ezaki, O. and Hirano, H. (1990). Erythrocyte/HepG2-type glucose transporter is concentrated in cells of blood-tissue barriers. Biochem. Biophys. Res. Commun. 173, 67-73.

Takata, K., Kasahara, T., Kasahara, M., Ezaki, O. and Hirano, H. (1992). Ultracytochemical localization of the erythrocyte/HepG2-type glucose transporter (GLUT1) in cells of the blood-retinal barrier in the rat. Invest. Ophthalmol. Vis. Sci. 33, 377-383.

Umino, Y., Everhart, D., Solessio, E., Cusato, K., Pan, J. C., Nguyen, T. H., Brown, E. T., Hafler, R., Frio, B. A., Knox, B. E. et al. (2006). Hypoglycemia leads to agerelated loss of vision. Proc. Natl. Acad. Sci. USA 103, 19541-19545.

Verhey, K. J., Hausdorff, S. F. and Birnbaum, M. J. (1993). Identification of the carboxy terminus as important for the isoform-specific subcellular targeting of glucose transporter proteins. J. Cell Biol. 123, 137-147.

Wieman, H. L., Horn, S. R., Jacobs, S. R., Altman, B. J., Kornbluth, S. and Rathmell, J. C. (2009). An essential role for the Glut1 PDZ-binding motif in growth factor regulation of Glut1 degradation and trafficking. Biochem. J. 418, 345-367.

Wilkin, G. P. and Wilson, J. E. (1977). Localization of hexokinase in neural tissue: light microscopic studies with immunofluorescence and histochemical procedures. $J$. Neurochem. 29, 1039-1051.

Winkler, B. S. (1981). Glycolytic and oxidative metabolism in relation to retinal function. J. Gen. Physiol. 77, 667-692.

Winkler, B. S., Starnes, C. A., Sauer, M. W., Firouzgan, Z. and Chen, S. C. (2004). Cultured retinal neuronal cells and Muller cells both show net production of lactate. Neurochem. Int. 45, 311-320.

Zhang, J. Z., Hayashi, H., Ebina, Y., Prohaska, R. and Ismail-Beigi, F. (1999). Association of stomatin (band 7.2b) with Glut1 glucose transporter. Arch. Biochem. Biophys. 372, 173-178.

Zhang, J. Z., Abbud, W., Prohaska, R. and Ismail-Beigi, F. (2001). Overexpression of stomatin depresses GLUT-1 glucose transporter activity. Am. J. Physiol. Cell Physiol. 280, C1277-C1283. 